\title{
A microscopic model for thin film spreading
}

\author{
Douglas B. Abraham ${ }^{1}$, Rodolfo Cuerno ${ }^{2}$, and Esteban Moro ${ }^{1}$ \\ ${ }^{1}$ Theoretical Physics, University of Oxford, 1 Keble Road OX1 3NP, United Kingdom \\ ${ }^{2}$ Dpto. de Matemáticas and GISC, Universidad Carlos III de Madrid, Avda. Universidad 30, 28911 Leganés, Spain
}

(February 1, 2008)

\begin{abstract}
A microscopic, driven lattice gas model is proposed for the dynamics and spatio-temporal fluctuations of the precursor film observed in spreading experiments. Matter is transported both by holes and particles, and the distribution of each can be described by driven diffusion with a moving boundary. This picture leads to a stochastic partial differential equation for the shape of the boundary, which agrees with the simulations of the lattice gas. Preliminary results for flow in a thermal gradient are discussed.
\end{abstract}

PACS numbers: 68.08.Bc, 68.15+e, 64.60.Ht, 05.70.Np

Spreading of involatile liquid drops on surfaces and fibers plays a significant role in many technologies, the efficient deployment of which requires detailed understanding of the underlying process [1,2]. Behavior at a macroscopic scale is accurately described by hydrodynamics [3], but experiments performed over the last 15 years or so have produced a surprise: in the case of complete wetting, the spreading drop, when examined on an atomic length scale in the direction normal to the substrate, is found to be preceded by a precursor film about one molecule thick; this can be followed laterally out to an extension of the order of $10^{7}$ molecules diameter. At the horizontal resolving power of the technique used (ellipsometry), the precursor film is found to be flat and homogeneous. Its radius advances with time as $\sqrt{t}$. Typical systems showing this class of behavior which can be investigated by ellipsometry are various silanes spreading over atomically flat $\operatorname{Si}(111)$ wafers with highly pure oxydised surfaces. With selected silanes, such systems even show dynamical layering with up to four superposed precursor films advancing as $\sqrt{t}$, the layer directly in contact with the substrate being much faster than the others. As Ball observed over a decade ago [1], at that time there was hardly even a formative theory of such phenomena. Such theory must capture the experimentally determined diffusive behavior and, at the same time, explain how an extremely viscous, involatile material is transported from the reservoir to the precursor edge.

A partial solution was first achieved by applying Molecular Dynamics to a droplet composed of spherical molecules with Lennard-Jones interactions $[$. The strength of these is adjusted to achieve sufficient involatility. Experimentally, the droplet is placed in contact with a substrate composed of much smaller units; this is therefore treated as a continuum for calculating the interaction of the spreading molecules with the substrate. Such a model shows a precursor film with the correct, diffusive behavior [4.5]. Monte Carlo (MC) lattice gas simulations in 3d 6, 16 confirm this, and suggest a dual mechanism of matter transport involving on the one hand particles located in a supernatant layer directly placed above the precursor film, and on the other, holes in the precursor film itself. At the same time, essentially all the configurational change occurs at the boundary of the drop. However, so far these ideas just constitute a picture without quantitative implications.

In this Letter, we introduce an associated $2 \mathrm{~d}$ driven Ising lattice gas model which can be simulated far more effectively. Extensive continuous time MC simulations are consistent with the $\sqrt{t}$ behavior found in all the experiments to which we refer [2]. They also allow us to formulate a probabilistic continuum model of independent hole and particle diffusion in the supernatant and precursor films, which accounts for the matter transfer between the reservoir and the precursor edge (PE). The motion of the latter in the driven lattice gas is then equivalent to a moving boundary in the continuum model. Starting from the constitutive equations of the moving boundary problem, we derive the effective dynamics of the $\mathrm{PE}$ as a stochastic partial differential equation. We thus obtain analytical predictions which are consistent both with experiments and with the original $2 \mathrm{~d}$ Ising model, and which can be extended to the asymptotic regime thus far unexplored both theoretically and experimentally.

We now describe our MC lattice gas simulations for this problem. At each cell on a square lattice $\boldsymbol{r}=(x, y, z)$ with unit lattice spacing, we define the ocuppation number $n(\boldsymbol{r}, t)$ to be 0 or 1 depending whether the cell is occupied or not. Moreover, we restrict $z$ to the values $z=1$ (precursor film) and $z=2$ (supernatant layer). The interaction energy for a given configuration is [6]

$$
\mathcal{H}=-J \sum_{|\boldsymbol{r}-\boldsymbol{s}|=1} n(\boldsymbol{r}, t) n(\boldsymbol{s}, t)-A \sum_{z=1,2} \frac{1}{z^{3}} n(\boldsymbol{r}, t),
$$

where the sums are over $r, s$ on the lattice. The last term is the van der Waals interaction with the continuum substrate characterized by a Hamaker constant $A>0$. We are interested in $J / k_{B} T$ large enough to achieve a high degree of involatility and $A / k_{B} T$ large enough to be in the complete wetting regime $[8]$. Continuous time MC Kawasaki dynamics are used [9] with initial conditions: (i) $n(\boldsymbol{r}, 0)=1$ for $y=1$, but with 


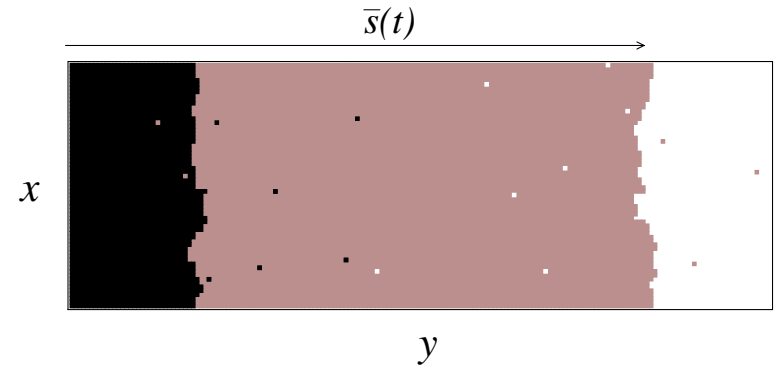

FIG. 1. Top view of a typical snapshot of our lattice gas model for $t=2 \times 10^{7} \mathrm{MC}$ units. Occupied cells in $z=1$ (precursor film) are in gray, while occupied cells in $z=2$ (supernatant film) appear in black. Non-colored cells are empty. Parameters used are $A=10, J=1, k_{B} T=1 / 3, L_{x}=128$.

any $x$ and for both $z=1$ and $z=2$; (ii) $n(\boldsymbol{r}, 0)=0$ for $y>1$. If, as a result of an allowed MC move at any later stage, $n(x, 1, z, t)=0$, then the implied vacancy is filled immediately from the putative reservoir droplet, $n(x, 1, z, t)=1$ being maintained in this sense for all $t>0$. Thus, the detailed evolution of the reservoir droplet is ignored. In reality, it would equilibrate relatively rapidly in profile by motion of particles near its surface and deflate by emission of supernatant particles and absorption of precursor holes [3]. The main advantage of the boundary condition we use, and a very significant one, is in computability. We believe our model to be new - it is two superposed Ising lattice gases driven from one edge.

A typical snapshot of the simulations is shown in Fig. 1. There is a compact film advancing in both the $z=1$ and $z=2$ layers, but the former (shown black) advances much faster that the latter (shown gray). It is easy to understand why this should be so: if a hole in the precursor film diffuses to lie beneath the compact film in $z=2$, at an appropriately selected update it will be filled by creating a hole in film above, which diffuses and may be trapped at the edge $y=1$, or at the supernatant film boundary, causing this film to shrink. The precursor advances because of emission of holes into itself, but also from the arrival of supernatant particles at its edge; such particles drop into the $z=1$ plane with concomitant Hamaker stabilization [10].

To study the dynamics of the PE, definitions of spinpercolative type are needed. Thus, the precursor film is the particle cluster $\mathcal{P}(t)$ in $z=1$ which is connected along nearest neighbor bonds to the boundary line $y=1$. The PE is at $y=h(x, t)$ for $x=1, \ldots, L_{x}$, where $h(x, t)$ is given by the maximum value of $y$ among those cells $(x, y)$ that belong to $\mathcal{P}(t)$. In Fig. 目 we present results for the dynamics of the PE: the velocity $V(t)=d \bar{s} / d t$ [panel (a)], where $\bar{s}(t)$ is the the mean PE displacement $\bar{s}(t)=\left(1 / L_{x}\right) \sum_{x=1}^{L_{x}} h(x, t)$, and the roughness $w^{2}\left(L_{x}, t\right)=\left(1 / L_{x}\right) \sum_{x=1}^{L_{x}}\left\langle\zeta^{2}(x, t)\right\rangle$ [panel (b)],

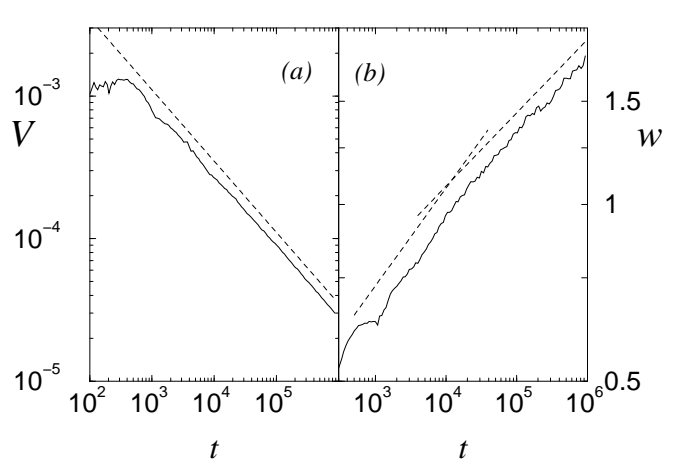

FIG. 2. Log-log plot of the instantaneous velocity [panel (a)] and roughness [panel (b)] of the PE. Solid lines are averages over 200 Monte Carlo realizations with $A=10, J=1$, $k_{B} T=1 / 3$, and $L_{x}=256$. All units are arbitrary. Dashed line in panel (a) depicts the $t^{-1 / 2}$ behavior, while those in panel (b) correspond to the $t^{1 / 6}$ and $t^{1 / 8}$ scaling behaviors.

where $\zeta(x, t) \equiv h(x, t)-\bar{s}(t)$ and $\langle\cdots\rangle$ stands for average over different MC runs. After a short initial transient $t=\tau_{0} \simeq 10^{3} \mathrm{MC}$ units (independent of $L_{x}$ ), we recover the law $\overline{\boldsymbol{s}}(t)=\gamma t^{1 / 2}$, with $\gamma$ a time independent constant. This is in clear qualitative agreement with the experimental results, and the confirmation of the universal $\sqrt{t}$ law is considerably more precise than in the 3 -d simulations [6]. On the other hand, for times longer than the initial transient $t>\tau_{0}$-before which the roughness oscillates due to the discreteness of the model and the flat initial condition on the $\mathrm{PE}-$, the $\mathrm{PE}$ displays kinetic roughening as reflected in the behavior $w\left(L_{x}, t\right) \sim t^{\beta}$ [11. The growth exponent $\beta$ seems to cross over from the value $\beta=1 / 6$ at intermediate times to $\beta=1 / 8$ at long times. Due to the restricted range of computationally accessible values of $L_{x}$, the roughness exponent $\alpha$ in the stationary state relation $w\left(L_{x}, \infty\right) \sim L_{x}^{\alpha} 11$. is more accurately extracted from the behavior of the structure factor $S(k, t)=\left\langle\zeta_{k}(t) \zeta_{-k}(t)\right\rangle$, where $\zeta_{k}(t)$ is the Fourier transform of $\zeta(x, t)$. For a rough interface, $S(k, t)$ is expected [11] to behave as $S(k, t) \sim k^{-(2 \alpha+1)}$ for long enough times. In Fig. 3(a) we show the structure factor of the PE measured for various times. As is clear from the figure, $S(k, t)$ tends to a scale invariant behavior at long times, characterized by a roughness exponent $\alpha=1 / 2$.

Inspection of typical runs shows a rather sparse distribution of particles [concentration $\left.c_{\mathrm{p}}(\boldsymbol{r}, t)\right]$ and holes [concentration $\left.c_{\mathrm{h}}(\boldsymbol{r}, t)\right]$, which attenuates very rapidly away from their respective sources [10]. Since the concentrations are typically low, the dynamics suggest to introduce an independent and non-interacting particle-hole diffusion model, with identical equations for particles and holes. We will treat the $r$ coordinate as a continuous variable. Thus, on $\mathcal{P}(t)$, we have 


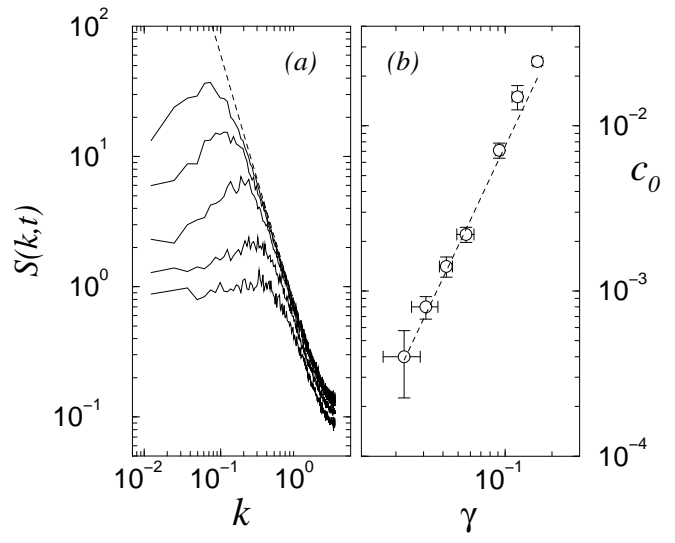

FIG. 3. (a) Structure factor of the PE for $t=1000,5248$, 29512, 165960, and $10^{6} \mathrm{MC}$ units (bottom to top). Each solid line is an average over $200 \mathrm{MC}$ realizations with $A=10$, $J=1, k_{B} T=1 / 3$, and $L_{x}=512$. Units are arbitrary. Dashed line is the exact asymptotic structure factor for the (discretized) Eq. (10), behaving as $S(k, \infty) \sim k^{-2}$ for small $k$. (b) Concentration of holes near the PE as a function of $\gamma$. Dashed line is the analytical prediction (月) and the circles are results of MC simulations from $k_{B} T=0.27$ up to $k_{B} T=0.5$, left to right. Other parameters are as in panel (a).

$$
\partial_{t} c_{\mathrm{h}}=D \Delta c_{\mathrm{h}}-\nabla \cdot \boldsymbol{q}
$$

where $\boldsymbol{q}(\boldsymbol{r}, t)$ is a noise term representing fluctuations in the hole diffusion current within $\mathcal{P}(t)$ [12]. The interface velocity along the outward normal $\boldsymbol{n}$ is given by

$$
V_{n}=2\left(D \nabla c_{\mathrm{h}}-\boldsymbol{q}\right) \cdot \boldsymbol{n}-B \nabla_{s}^{2} \kappa-\nabla_{s} \cdot \boldsymbol{p} .
$$

The first term on the right in (3) comes from diffusion of holes away from the PE. The prefactor two reflects the dual rôle of particles and holes and that both have the same diffusion constant. The second term represents surface diffusion due to the tendency for particles/holes to move along the interface from regions of negative to positive curvature, the constant $B$ being proportional to the interface concentration of mobile species. Here $\kappa$ is the mean curvature. Finally, $\boldsymbol{p}(\boldsymbol{r}, t)$ is a noise term describing fluctuations in the interface diffusion current along the precursor edge [13]. The concentration obeys also the boundary condition $c_{\mathrm{h}}(x, 0, t)=0$ and the GibbsThompson condition [14]

$$
\left.c_{\mathrm{h}}(\boldsymbol{r}, t)\right|_{y=h(x, t)}=c_{0}+\Gamma \kappa,
$$

where $c_{0}$ is the average concentration of holes at the precursor edge and $\Gamma=c_{0} \sigma / k_{B} T$, where $\sigma$ is the surface tension, which to leading order is taken to be isotropic.

This moving boundary problem presents formidable obstacles to direct attack. Neglecting the noise terms $\boldsymbol{q}$ and $\boldsymbol{p}$, assuming a flat interface, and averaging over $x$, a classical Stefan problem [15] is recovered for the mean hole concentration $\bar{c}(y, t)$. On $\mathcal{P}(t), \bar{c}(y, t)$ satisfies the diffusion equation $\partial_{t} \bar{c}=D \partial_{y}^{2} \bar{c}$, but now with far simpler boundary conditions $\left.\bar{c}(y, t)\right|_{y=\bar{s}(t)}=c_{0}, \bar{c}(0, t)=0$ and

$$
\frac{d \bar{s}}{d t}=\left.2 D \partial_{y} \bar{c}\right|_{y=\bar{s}(t)} \text {. }
$$

This problem admits a similarity solution [15 in which $\bar{s}(t)=\gamma t^{1 / 2}$, and

$$
\bar{c}(y, t)=\frac{\gamma}{4 D} \int_{0}^{y / t^{1 / 2}} e^{\left(\gamma^{2}-u^{2}\right) / 4 D} d u
$$

where $\gamma$ is the unique solution of

$$
c_{0}=\frac{\gamma}{4 D} \int_{0}^{\gamma} e^{\left(\gamma^{2}-u^{2}\right) / 4 D} d u
$$

Equation (7) then gives a relation between $\gamma$ and $c_{0}$. Now, both $\gamma$ and $c_{0}$ can be read off from the simulations, with different choices of temperature giving different $\left(c_{0}, \gamma\right)$ points which are compared in Fig. 3(b) with the predictions of (7). The way the simulations of the lattice gas are set up implies that, for comparison with the diffusive system, we should have $D=1 / 3$. The excellent agreement in Fig. 3(b) furnishes very significant support for our continuum model.

The spatio-temporal fluctuations of the PE, expressed by $w\left(L_{x}, t\right)$ and $S(q, t)$ in Figs. 2(b) and 3(a), respectively, require full implementation of the boundary conditions (3) and (4), a formidable mathematical problem. Progress can be made when $\gamma$ is small [see Fig. 3(b)] by (i) setting $\partial_{t} c_{\mathrm{h}}=0$ for $y<h(x, t)$, the quasistationary approximation [16]; (ii) perturbing around the spatially-averaged solution given by (6) and (7) [17]: we expand Eq. (3) to quadratic order in the fluctuation variable $\zeta(x, t)=h(x, t)-\bar{s}(t)$ describing the precursor edge -taking the noise terms to be of the same order as $\zeta(x, t)$ [12]-, to obtain

$$
\begin{aligned}
\frac{\partial \zeta_{k}}{\partial t}= & -\left[D|k|\left(\Gamma k^{2}+\frac{c_{0}}{\gamma t^{1 / 2}}\right) \operatorname{coth}\left(|k| \gamma t^{1 / 2}\right)+B k^{4}\right] \zeta_{k} \\
& +\frac{V(t)}{2} \mathcal{F}_{k}\left[\left(\partial_{x} \zeta\right)^{2}\right]+\eta_{k}(t),
\end{aligned}
$$

where $\mathcal{F}_{k}[f(x)]$ is the Fourier transform of $f(x)$, and $\eta_{k}(t)$ is a white noise with correlations [19]

$$
\begin{aligned}
\left\langle\eta_{k}(t) \eta_{k^{\prime}}\left(t^{\prime}\right)\right\rangle & =2 c_{0} \delta_{k,-k^{\prime}} \delta\left(t-t^{\prime}\right) \\
& \times\left\{D\left[1+\frac{|k|^{2} \gamma t^{1 / 2}}{\sinh ^{2}\left(|k| \gamma t^{1 / 2}\right)}\right]+\frac{B}{\Gamma} k^{2}\right\} .
\end{aligned}
$$

In Eq. (8) the linear terms stabilize PE fluctuations, while the nonlinear term is of the Kardar-Parisi-Zhang (KPZ) type [20], with a coefficient $V(t)=\gamma / t^{1 / 2}$ that decays in time. In the asymptotic regime $t \rightarrow \infty$, and neglecting terms proportional to $c_{0}, \Gamma \sim \mathcal{O}\left(\gamma^{2}\right)$, Eqs. (8) and (9) reduce to the conserved linear equation 


$$
\frac{\partial \zeta_{k}}{\partial t}=-B k^{4} \zeta_{k}+\eta_{k}
$$

with noise correlations $\left\langle\eta_{k}(t) \eta_{k^{\prime}}\left(t^{\prime}\right)\right\rangle=2\left(c_{0} B / \Gamma\right) k^{2} \delta_{k,-k^{\prime}}$ $\delta\left(t-t^{\prime}\right)$. It is easy to solve this equation exactly and obtain $\beta=1 / 8$ and $\alpha=1 / 2$, which provide accurate fits at long times for the behavior of the roughness [Fig. 2(b)] and the structure factor [Fig. 3(a)], respectively. Moreover, the behavior of $S(k, t)$ at long but finite times [e.g., $t=10^{6} \mathrm{MC}$ units in Fig. 3(a)] is also consistent with that predicted by Eq. (10). For sufficiently long times, the rate of arrival of particles becomes essentially zero. In this case, emission of particles to the exterior of the PE and holes to the interior are in balance, so the mean interface is stationary. In such a situation, up to order $\mathcal{O}\left(\gamma^{2}\right)$, the only fluctuations on the PE are due to the conserved surface diffusion current, which is precisely described by Eq. (10). However, for intermediate times (e.g., $t \lesssim 10^{5}$ $\mathrm{MC}$ units), the behavior of the fluctuations deviates from that predicted by Eq. (10), see Figs. 2(b) and 3(a). A plausible explanation is that the KPZ term in Eq. (8) is still non-negligible, in which case it should dominate the large scale properties of the PE. Using results for the KPZ equation [11,21, the roughness should then be given in this time regime by $w \sim[V(t) t]^{1 / 3} \sim t^{1 / 6}$, again in rather good agreement with the numerical results for the lattice gas, see Fig. 2(b).

In summary, using simulations of our new driven lattice gas model we have (i) recaptured the $\sqrt{t}$ universal mean precursor displacement law; (ii) shown that the spatiotemporal fluctuations of the PE demonstrate dynamical scaling; (iii) shown that the mechanisms of matter transport from the reservoir droplet to the PE involve low density of particles and holes, described by diffusion equations with moving boundaries; (iv) derived a stochastic partial differential equation for the $\mathrm{PE}$ which brings in the fluctuation regimes found previously in a natural way, and which tends asymptotically to a Langevin description for a free interface with conserved dynamics as in (10), possibly observable experimentally, even though it presents a KPZ behavior at intermediate times. In preliminary work, we have investigated our lattice gas model for a single layer $(z=1)$ in a thermal gradient, finding a mean spatial displacement growing linearly in time, generated by biased hole diffusion. This has relevance for microfluidics [22].

We thank A. Sánchez for participation in the early stages of this work, and M. Castro for discussions. E.M. acknowledges the EU fellowship No. HPMF-CT-20000487. This research has been supported by EPSRC (UK) Grant No. GR/M04426 and by DGES (Spain) grants Nos. HB1999-0018 and BFM2000-0006.
[1] P.M. Ball, Nature 338, 624 (1989); K. Kaski, Europhys. News 26, 23 (1995).

[2] L. Léger, M. Erman, A.M. Guinet-Picard, D. Ausserre, and C. Strazielle, Phys. Rev. Lett. 60, 2390 (1988); F. Heslot, N. Fraysse, and A.M. Cazabat, Nature 338, 640 (1989); F. Heslot, A.M. Cazabat, and P. Levinson, Phys. Rev. Lett. 62, 1286 (1989); M.L. Forcada and C.M. Mate, Nature 363, 527 (1993).

[3] P.G. de Gennes, Rev. Mod. Phys. 57, 827 (1985).

[4] J.A. Nieminen, D.B. Abraham, M. Karttunen, and K. Kaski, Phys. Rev. Lett. 69, 124 (1992).

[5] J. De Coninck, U. D'Ortona, J. Koplik, and J.R. Banavar, Phys. Rev. Lett. 74, 928 (1995); S. Bekink, S. Karaborni, G. Verbist, and K. Esselink, ibid. 76, 3766 (1996).

[6] A. Lukkarinen, K. Kaski, and D.B. Abraham, Phys. Rev. E 51, 2199 (1995).

[7] S.F. Burlatsky, G. Oshanin, A.M. Cazabat, and M. Moreau, Phys. Rev. Lett. 76, 86 (1996); S.F. Burlatsky, G. Oshanin, A.M. Cazabat, M. Moreau, and W.P. Reinhardt, Phys. Rev. E 54, 3832 (1996).

[8] S. Dietrich, in Phase Transitions and Critical Phenomena, edited by C. Domb and J.L. Lebowitz, Vol. 12, p. 1 (Academic Press, New York, 1988).

[9] Kinetic $\mathrm{MC}$ is implemented with hopping rate $\tau=$ $\nu \exp \left[-\Delta \mathcal{H} / k_{B} T\right]$, where $\Delta \mathcal{H}$ is the energy change in the move and $\nu$ is the attempt frequency, which we take equal to the inverse number of destination sites thereby fixing our time scale. See M.E.J. Newman and G.T. Barkema, Monte Carlo methods in Statistical Physics (Oxford University Press, New York, 1999).

[10] There is also emission of particles from the precursor edge into the free space ahead of it. This gas of particles is close to equilibrium with the interface and thus, its effect will be neglected in Eq. (3).

[11] A.-L. Barabási and H.E. Stanley, Fractal Concepts in Surface Growth, Cambridge University Press, Cambridge (1995); J. Krug, Adv. Phys. 46, 139 (1997).

[12] A. Karma and C. Misbah, Phys. Rev. Lett. 71, 3810 (1993).

[13] L.-H. Tang and T. Nattermann, Phys. Rev. Lett. 66, 2899 (1991); R. Cuerno and M. Castro (to be published).

[14] In Eq. (4) fluctuations [12 have been suppressed due to the absorbing boundary condition implicit in Eq. (3).

[15] J. Crank, Free and Moving Boundary Problems (Clarendon Press, Oxford, 1987).

[16] J.S. Langer, Rev. Mod. Phys. 52, 1 (1980).

[17] S.H. Doole, Mathl. Comput. Modelling 23, 55 (1996).

[18] E. Moro, R. Cuerno, and D.B. Abraham, in preparation.

[19] The correlations for $\eta_{k}(t)$ originate from those for $\boldsymbol{q}$ in Eq. (2) and $\boldsymbol{p}$ in Eq. (3). A local equilibrium hypothesis determines these to be $\left\langle q_{i}(\boldsymbol{r}, t) q_{j}\left(\boldsymbol{r}^{\prime}, t^{\prime}\right)\right\rangle=2 D c_{0} \delta_{i j} \delta(\boldsymbol{r}-$ $\left.\boldsymbol{r}^{\prime}\right) \delta\left(t-t^{\prime}\right)$ and $\left\langle p_{i}(\boldsymbol{r}, t) p_{j}\left(\boldsymbol{r}^{\prime}, t^{\prime}\right)\right\rangle=2\left(B c_{0} / \Gamma\right) \delta_{i j} \delta(\boldsymbol{r}-$ $\left.r^{\prime}\right) \delta\left(t-t^{\prime}\right)$, see 12,13.

[20] M. Kardar, G. Parisi, and Y.-C. Zhang, Phys. Rev. Lett. 56, 889 (1986).

[21] E. Hernández-García, T. Ala-Nissila, and M. Grant, Europhys. Lett. 21, 401 (1993).

[22] D.E. Kataoka and S.M. Troian, Nature 402, 794 (1999). 